\title{
Evolución histórica de la calidad biológica del agua en la cuenca del Ebro (España) (1990-2005)
}

\author{
Javier Oscoz ${ }^{1, *}$, Concha Durán ${ }^{2}$, Miriam Pardos ${ }^{2}$, Joaquín Gil ${ }^{3}$ y Ana Viamonte ${ }^{4}$ \\ ${ }^{1}$ Departamento de Zoología y Ecología, Facultad de Ciencias, Universidad de Navarra, Apdo. 177, E-31080, \\ Pamplona. \\ 2 Área de Calidad de Aguas, Confederación Hidrográfica del Ebro, $\mathrm{P}^{\mathrm{o}}$ de Sagasta 24-28, 50071, Zaragoza \\ ${ }^{3}$ Applus+ Medioambiente Alicante, Polígono Industrial "Las Atalayas", C/ Dracma, 16-18, C.P. 03114, Alicante \\ ${ }^{4}$ Applus+ Medioambiente Aragón, Juan Carlos I, 3 bajo. C.P. 50006, Zaragoza \\ * Corresponding author: joscoz@alumni.unav.es
}

Recibido: $13 / 7 / 07 \quad$ Aceptado: $11 / 10 / 07$

\begin{abstract}
Historical evolution of the biological water quality in the Ebro Basin (Spain) (1990-2005)

From 1990 to 2005 the Ebro River Basin has been monitored for biological water quality. The number of sampling points analysed has progressively increased along this period. The observed results show that water quality has improved with respect to the initial time of the study. In 2005, almost $80 \%$ of the studied sampling stations reached a "Good" or "Very good" water quality level according to the IBMWP biotic index. In 2005 most of the sampling points had a higher IBMWP value compared to its historic mean value. Similar results were found in relation to the ecological status in 2005 in this basin when compared to the corresponding historical mean values at each sampling point.
\end{abstract}

Key words: Biological water quality, historical evolution, macroinvertebrates, biotic index, IBMWP, Ebro River Basin.

\section{RESUMEN}

Evolución histórica de la calidad biológica del agua en la cuenca del Ebro (España) (1990-2005)

Entre 1990 y 2005 se han realizado estudios de la calidad biológica de las aguas fluviales en la cuenca del río Ebro. A lo largo de este periodo se ha incrementado el número de estaciones de muestreo estudiadas, habiéndose además observado una mejora general de la calidad del agua respecto a la época inicial del estudio. En el año 2005, casi el $80 \%$ de los tramos estudiados alcanzaron una clase de calidad "Buena" o "Muy Buena" según el índice IBMWP. La mayoría de las estaciones tuvieron en el año 2005 un valor de IBMWP más alto respecto a su media histórica. Resultados similares se hallaron en relación al estado ecológico en 2005 respecto al correspondiente a los valores medios históricos en cada estación.

Palabras clave: Calidad biológica del agua, evolución histórica, macroinvertebrados, Indice biótico, IBMWP, Cuenca del Ebro.

\section{INTRODUCCIÓN}

El crecimiento económico en los países desarrollados puede conllevar una serie de procesos, como la contaminación de las aguas y la alteración del hábitat en los ecosistemas acuáticos, que provocan la desaparición de especies y afec- tan además a la calidad del agua. Este deterioro de la calidad puede dificultar la utilización del agua como recurso, ya sea para consumo humano, para riego o para otras actividades. Por ello se comprende que la evaluación de la calidad del agua en un ecosistema acuático es una necesidad, pues así se puede determinar qué ti- 
pos de aprovechamiento pueden realizarse o qué factores influyen en el mismo, para proponer posibles actuaciones de mejora.

En la determinación de la calidad del agua tradicionalmente se han utilizado parámetros fisicoquímicos, si bien el coste y la laboriosidad que representa analizar todos los posibles contaminantes hacen que en la práctica sólo unos pocos de ellos se analicen. La información que proporcionan estos análisis es valiosa y precisa, pero los resultados que ofrecen se limitan al momento de la toma de la muestra, lo que ha llevado a prestar una mayor atención a los indicadores biológicos, que son un reflejo, entre otros aspectos, de las condiciones fisicoquímicas tanto en el momento del muestreo como las sucedidas en un pasado reciente. Por ello, en los estudios sobre calidad de aguas, los análisis biológicos se han convertido en un importante complemento de los análisis fisicoquímicos, no debiendo considerarse como métodos excluyentes (García de Jalón et al., 1980; Alba-Tercedor, 1996).

Esta complementariedad e importancia de los análisis biológicos se recoge en la Directiva 2000/60/CE (Directiva Marco del Agua, DMA) (D.O.C.E., 2000), donde se demanda la utilización de métodos biológicos para estimar no ya la calidad del agua, sino el estado ecológico de los ríos. El principal objetivo de la DMA es conseguir un buen estado ecológico para los ecosistemas acuáticos de la Comunidad Europea antes del año 2015, protegiéndolos, evitando su deterioro y promoviendo el uso sostenible de las aguas. La mencionada DMA establece que los indicadores biológicos (fitoplancton, macrófitos, organismos fitobentónicos, fauna bentónica de invertebrados y fauna ictiológica) han de ser los que determinen en última instancia el estado ecológico de una masa de agua. Para ello el estado ecológico se define mediante una comparación de la comunidad biológica observada con la que presenten tramos casi inalterados considerados de referencia. De cara a establecer dichos puntos de referencia, tanto si se utiliza para ello una base espacial o una modelización, el conocimiento de los datos históricos previamente existentes en una cuenca hidrográfica es una herramienta más a utilizar.
Desde 1990 se vienen realizando en la cuenca del Ebro estudios sobre la calidad del agua mediante el uso de macroinvertebrados bentónicos, aplicando el índice BMWP' (Alba-Tercedor y Sánchez-Ortega, 1988) en los primeros años de estudio, y su versión posterior, denominada IBMWP (Alba-Tercedor, 1996; Alba-Tercedor et $a l .$, 2002), en los últimos años del mismo. Dichos estudios fueron iniciados por el Centro de Estudios y Experimentación de Obras Públicas (CEDEX) en los años 1990-1993. Posteriormente, la Oficina de Planificación Hidrológica de la Confederación Hidrográfica del Ebro se encargó de la recogida de datos, y en los últimos años ha sido el Área de Calidad de Aguas quien ha realizado estos estudios. A lo largo de los años se ha ido ampliando la red de control hasta llegar a tener más de 500 estaciones de muestreo repartidas por toda la cuenca del Ebro, que constituían la denominada Red de Control de Variables Ambientales (RCVA) (Fig. 1), actualmente en fase de redefinición con el fin de adaptarse a los requisitos de la DMA.

En el presente estudio se exponen los datos existentes sobre la calidad del agua determinada mediante índices bióticos basados en macroinvertebrados bentónicos en la cuenca del Ebro entre los años 1990 y 2005, observando la evolución que ha existido a lo largo de este periodo, y analizando con más detalle la situación hallada en la campaña del año 2005.

\section{ÁREA DE ESTUDIO}

La cuenca del río Ebro (Fig. 1) tiene una superficie de más de $85000 \mathrm{Km}^{2}$, abarcando territorio de nueve comunidades autónomas y 18 provincias, en las que se encuentran unos 1600 municipios con más de 5000 localidades, y en las que existen cerca de 2800000 habitantes. En la cuenca existen 347 ríos principales que suman en longitud más de $12000 \mathrm{Km}$. De acuerdo a la caracterización de los Tipos fluviales establecida por el Ministerio de Medio Ambiente para la implementación de la DMA en España, en cumplimiento de su Anexo II, en esta cuenca se encuentran ocho Tipos Fluvia- 


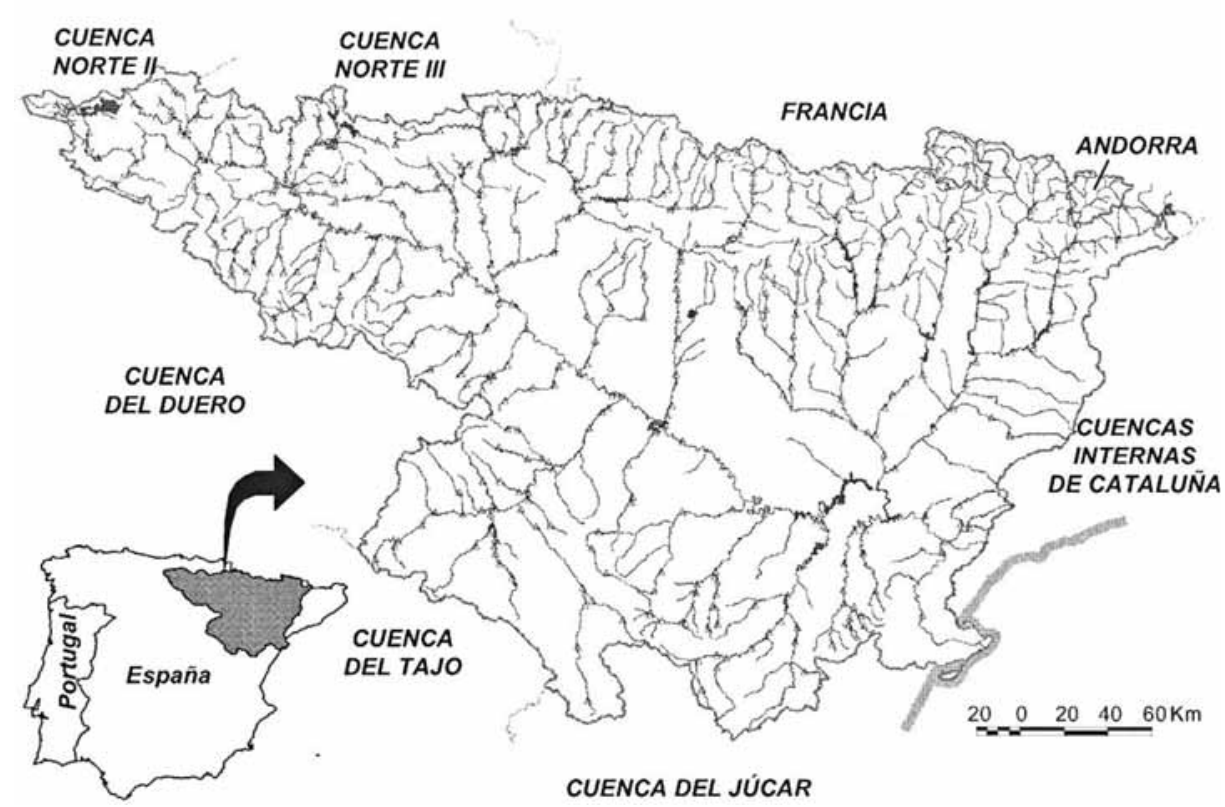

Figura 1. Área de estudio y localización de las estaciones de muestreo en la cuenca del Ebro. Study area and location of the sampling stations in the Ebro Basin.

les diferentes con el siguiente porcentaje de representación respecto al total de kilómetros de río existentes en la cuenca: 1) Ríos mineralizados de baja montaña mediterránea $(20.5 \%), 2)$ Ríos de montaña mediterránea silícea $(2.4 \%), 3$ ) Ríos de montaña mediterránea calcárea (31.1\%), 4) Ejes mediterráneos continentales poco mineralizados $(6.4 \%)$, 5) Ejes mediterráneos continentales mineralizados $(1.0 \%)$, 6) Grandes ejes en ambiente mediterráneo (4.3\%), 7) Ríos de montaña húmeda calcárea $(25.8 \%)$ y 8$)$ Ríos de alta montaña $(8.6 \%)$.

\section{MATERIAL Y MÉTODOS}

Para la obtención de las muestras de macroinvertebrados, se han seguido los protocolos descritos en las diferentes versiones del índice aplicado desde su primera descripción en España (Alba-Tercedor y Sánchez-Ortega, 1998; AlbaTercedor, 1996; Jáimez-Cuellar et al., 2002). En general, se ha utilizado una manga de recolección con una luz de malla de $0.5 \mathrm{~mm}$, muestreándose todos los hábitats diferentes previamente identi- ficados en el tramo, de acuerdo a lo expresado en los protocolos de aplicación del índice biótico citados anteriormente. Para evitar la obstrucción de la malla durante el periodo de muestreo se vacía cada poco tiempo el contenido de la red. La muestra se fija in situ en una solución de formaldehido al $4 \%$. El recipiente debidamente etiquetado se traslada al laboratorio para su posterior estudio. En el laboratorio las muestras se lavan con agua, filtrándose con tamices de diferente luz a fin de retirar parte del material inerte y concentrar la población de macroinvertebrados. El filtrado se conserva en frascos de cristal con alcohol al $70 \%$ debidamente etiquetado. Cada taxón se identifica mediante lupa binocular hasta nivel de familia, ya que este es el nivel taxonómico requerido para calcular el índice biótico empleado, siendo un indicador fidedigno de las condiciones ambientales (Graca et al., 1995; Dolédec et al., 2000).

Para determinar las clases de calidad del índice biótico se han utilizado los rangos de calidad establecidos en cada versión del índice utilizado (BMWP': Alba-Tercedor \& Sánchez-Ortega, 1998; IBMWP: Alba-Tercedor et al., 2002), a la 


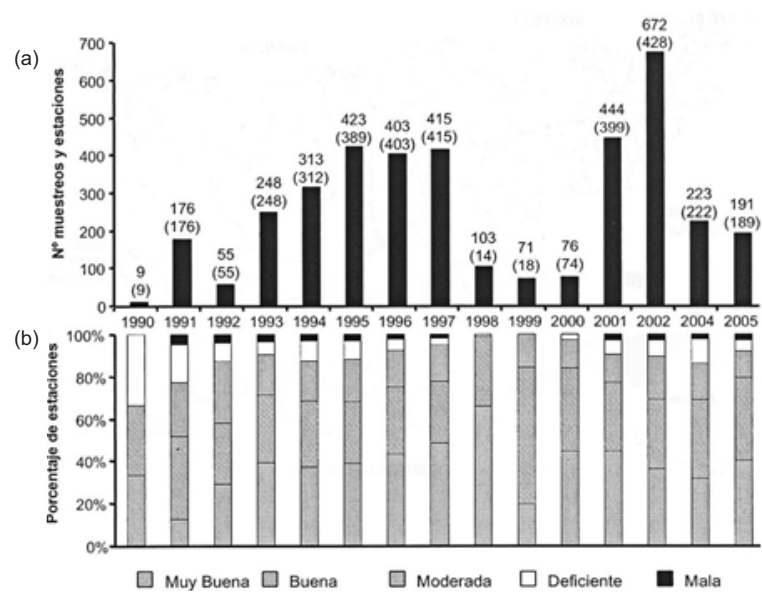

Figura 2. Número de muestras, estaciones analizadas (entre paréntesis) y porcentaje de cada clase de calidad del agua hallada en la cuenca del Ebro (1990-2005). Number of samples, sampling stations analysed (between brackets) and percentage of each water quality class found in the Ebro Basin (19902005).

espera de que finalicen los trabajos de definición de las condiciones de referencia según la DMA, en los que se espera disponer de un número adecuado de estaciones de referencia que permita establecer una escala de clasificación de estado ecológico para cada ecotipo fluvial.

\section{RESULTADOS}

\section{Evolución de la calidad del agua (1990-2005)}

El número de muestras y estaciones estudiadas anualmente en la cuenca del Ebro en el periodo 1990-2005 han ido en aumento desde los años iniciales, analizándose en los últimos años (salvo en el periodo 1998-2000) un número de estaciones cercano o superior a 200 (Fig. 2a). Respecto a la campaña de 2005 , hay que señalar que aunque se había previsto analizar un total de 227 estaciones, debido a la sequía sufrida dicho año sólo se pudieron tomar y analizar finalmente 191 muestras. Los resultados obtenidos respecto a la calidad de las aguas en estos años se muestran en la figura $2 b$, pudiendo observarse la existencia de una mejora general de la calidad del agua en la cuenca del río Ebro en este periodo. El porcentaje de estaciones que alcanzaron al menos el estado ecológico "Bueno" difirió significativamente en el periodo de estudio $\left(\chi^{2}=119.1 ; p<0.01\right)$, observándose una relativa estabilidad en el porcentaje de estaciones que alcanzaron al menos dicho estado en la mayor parte de los últimos años. Si se consideran sólo las últimas cuatro campañas, en las cuales teóricamente el índice está mejorado en su protocolo de aplicación, también se encontró una diferencia significativa en el porcentaje de estaciones con un estado ecológico al menos "Bueno" $\left(\chi^{2}=14.3 ; p<0.01\right)$. Sin contar el periodo 1998-2000, en el que el número de estaciones y muestras analizadas fue bastante menor que en casi todas las restantes campañas, el mayor porcentaje de estaciones con calidad del agua "Buena" o superior se obtuvo en el año 2005.

Si se desglosan los resultados obtenidos en el periodo de estudio diferenciando los ocho tipos fluviales (Fig. 3) se puede observar que parece haber existido en general un incremento en el porcentaje de estaciones que alcanzaban niveles de calidad "Buena" о "Muy Buena", especialmente en los tipos fluviales localizados en tramos medios y bajos, lo que indicaría que la calidad del agua en este periodo ha mejorado a lo largo del tiempo. Sólo en el tipo fluvial Ejes mediterráneos continentales mineralizados se ha mantenido a lo largo de los años una calidad por debajo de "Buena" en casi todos los estudios realizados. Si se analiza el porcentaje de estaciones que alcanzaron al menos el estado ecológico "Bueno" en todo el periodo de estudio se encuentra que sólo en los ecotipos Ríos de montaña mediterránea silícea y Ejes continentales mediterráneos mineralizados éste no difirió significativamente $\left(\chi^{2}=0.0 ; p>0.05\right.$ $\mathrm{y} \chi^{2}=5.9 ; p>0.05$ respectivamente). En el resto de los ecotipos dicha diferencia fue significativa (Ríos mineralizados de baja montaña mediterránea $\left(\chi^{2}=44.9 ; p<0.01\right)$, Ríos de montaña mediterránea calcárea $\left(\chi^{2}=60.8\right.$; $p<0.01)$, Ejes mediterráneos continentales poco mineralizados $\left(\chi^{2}=36.6 ; p<0.01\right)$, Grandes ejes en ambiente mediterráneo $\left(\chi^{2}=48.6\right.$; $p<0.01)$, Ríos de montaña húmeda calcárea $\left(\chi^{2}=39.9 ; p<0.01\right)$ y Ríos de alta montaña $\left(\chi^{2}=38.4 ; p<0.01\right)$. Sin embargo si sólo se analizaban las últimas cuatro campañas sólo se 
Rios mineralizados de baja montaña mediterránea

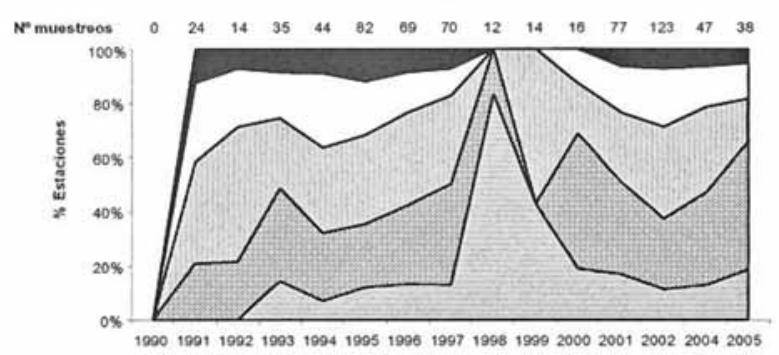

Rios de montaña mediterránea calcárea

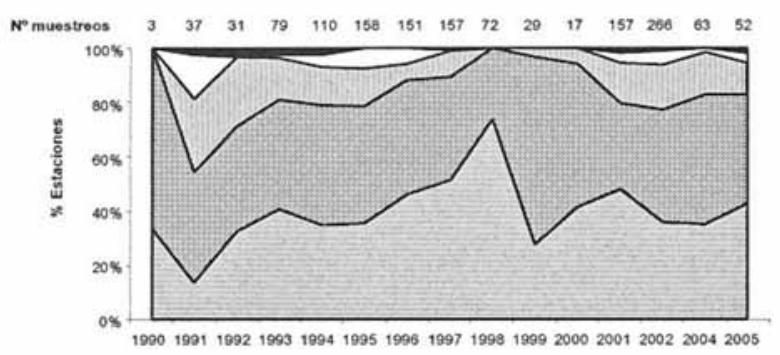

Ejes continentales mediterráneos mineralizados
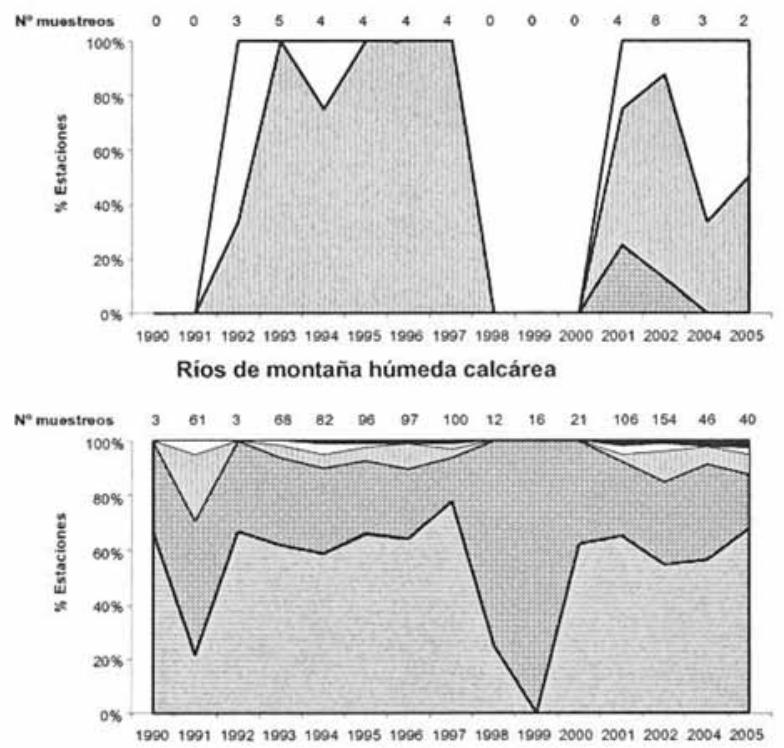

Rios de montaña mediterránea silicea

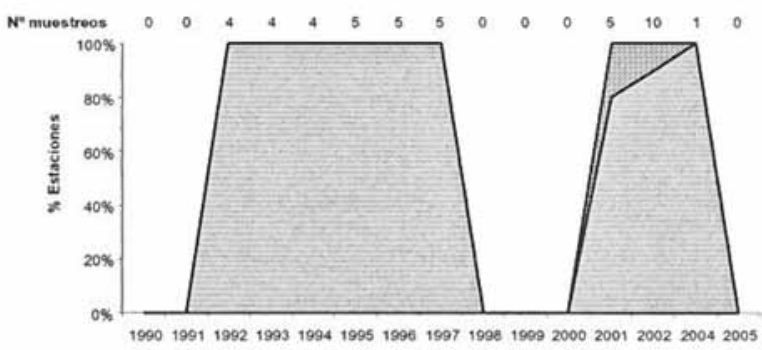

Ejes mediterráneos continentales poco mineralizados

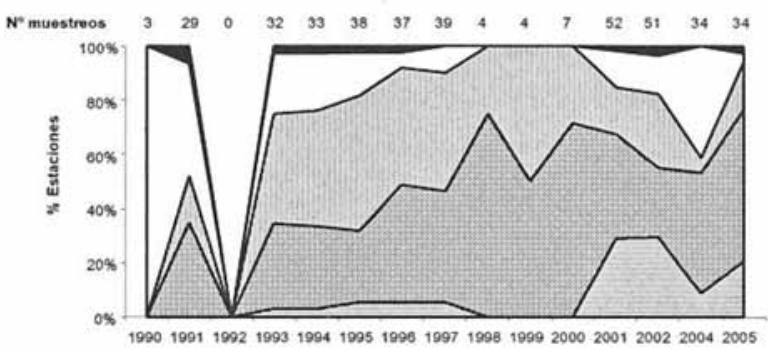

Grandes ejes en ambiente mediterráneo
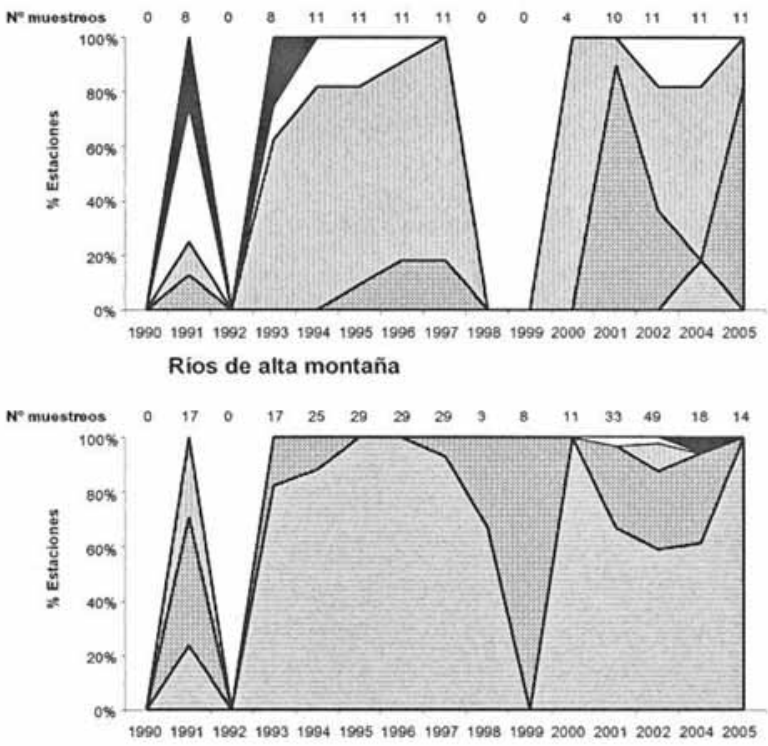

Muy Buena

Buena

Moderada

Deficiente

Mala

Figura 3. Número de estaciones analizadas y porcentaje de cada clase de calidad del agua según el índice IBMWP (antiguo BMWP' $^{\prime}$ ) obtenida en la cuenca del Ebro en cada tipo fluvial (1990-2005). Number of sampling stations analysed and percentage of each water quality class according to the IBMWP index (formerly BMWP') in each fluvial type in the Ebro Basin (1990-2005).

mantuvieron diferencias significativas en el porcentaje de estaciones con estado ecológico al menos "Bueno" en los ecotipos Ríos mineralizados de baja montaña mediterránea $\left(\chi^{2}=10.3 ; p<\right.$ $0.05)$ y Grandes ejes en ambiente mediterráneo $\left(\chi^{2}=15.76 ; p<0.01\right)$. 


\section{Calidad del Agua (año 2005)}

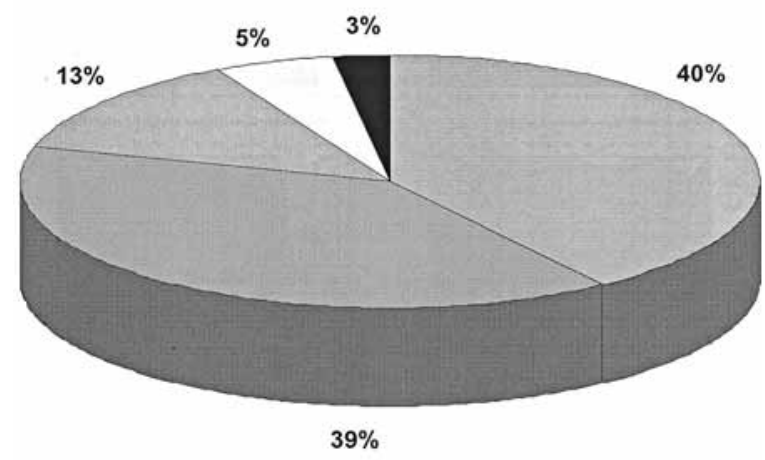

Muy Buena
Moderada Deficiente

Figura 4. Porcentaje de estaciones en cada clase de calidad del agua obtenida en la cuenca del Ebro en el año 2005. Percentage of sample stations in each water quality class in the Ebro Basin in 2005.

\section{Calidad del agua en el año 2005}

Respecto a los resultados obtenidos en la campaña del año 2005, casi el $80 \%$ de las estaciones analizadas alcanzaron una clase de calidad al menos "Buena" (Fig. 4), tal y como requiere la DMA como objetivo para el año 2015. Comparando los resultados obtenidos en las estaciones analizadas el año 2005 con los valores medios históricos hallados en las mismas a lo largo del periodo 1990-2005, y adoptando el criterio de que existía una mejora o un empeoramiento del valor del índice cuando la diferencia era mayor que 10 puntos (lo que implicaría que al menos han debido aparecer o desaparecer dos taxones), se encontró que más del $65 \%$ de las estaciones tuvieron un valor del IBMWP mayor que su media histórica. Casi el $25 \%$ de las estaciones tuvieron valores similares, mientras que menos del $10 \%$ tuvieron un valor peor. Si lo que se compara es la clase de calidad hallada en cada estación en la campaña de 2005 con aquella clase a la que correspondería el valor medio histórico se encontró que casi el $50 \%$ de los puntos analizados mejoraron su clase de calidad, algo más del $40 \%$ tuvieron una calidad similar, mientras que menos del $10 \%$ de las estaciones vieron reducida su clase de calidad. Si se analiza el

valor del índice IASPT hallado en el año 2005 respecto a la media histórica de dicho índice en el periodo 1990-2005, se encontró que un $60 \%$ de las estaciones analizadas aumentaron su valor, lo que implicaría que en dichas estaciones la presencia de organismos con menor tolerancia a la polución habría aumentado respecto a la de organismos con menores requisitos en cuanto a la calidad de las aguas.

En la figura 5 se muestran los resultados de calidad biológica del agua hallados en la campaña del año 2005 en los diferentes tipos fluviales estudiados. Se observa que sólo en las estaciones del tipo fluvial -Ejes mediterráneos continentales mineralizados- no se alcanza una calidad "Buena", mientras en el resto de tipos fluviales la mayoría de las estaciones analizadas alcanzaron una calidad al menos "Buena". Si se compara, al igual que se ha hecho antes, los resultados obtenidos en las estaciones analizadas considerando los distintos tipos fluviales con los valores medios históricos (Fig. 6) se observa que en el año 2005 se obtuvieron valores mayores en el índice biótico en la mayoría de los tipos fluviales. Solamente en el tipo fluvial -Ejes mediterráneos continentales mineralizados- no se hallaron valores superiores a la media histórica, sino que fueron similares. Unas pocas estaciones de muestreo tuvieron un valor menor que su media histórica en algunos tipos fluviales. Si se compara la clase de calidad hallada en el año 2005 con la clase correspondiente al valor medio histórico se observan resultados similares (Fig. 6). Por su parte, comparando los valores medios históricos del índice IASPT con los obtenidos en la campaña del 2005 se observa que dicho año se mejoró el valor del índice en la mayoría de las estaciones de los distintos ecotipos fluviales (Tabla 1), siendo esto sólo al contrario en el ecotipo -Ríos de alta montaña-.

\section{DISCUSIÓN}

\section{Evolución de la calidad del agua (1990-2005)}

El incremento general en el número de muestreos realizados y estaciones muestreadas a lo largo 


\section{Calidad del agua por tipos fluviales (año 2005)}

\section{R. mineralizados baja montaña mediterránea}

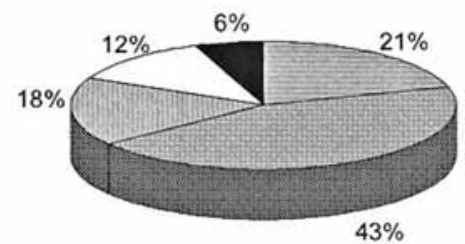

Ejes medit. continentales mineralizados

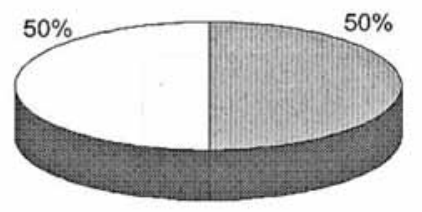

Rios de alta montaña

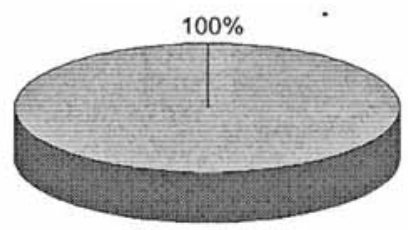

Ríos de montaña mediterránea calcárea

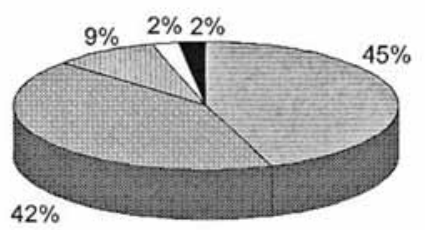

Grandes ejes en ambiente mediterráneo

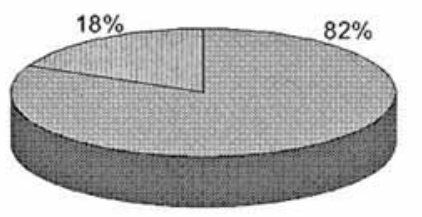

Ejes medit. continentales poco mineralizados

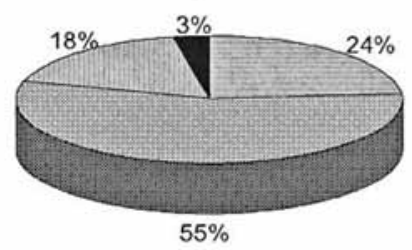

Ríos de montaña húmeda calcárea

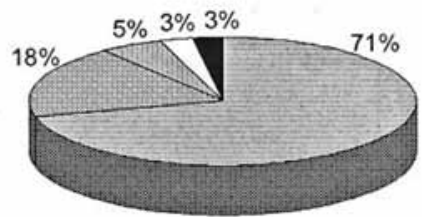

Figura 5. Porcentaje de cada clase de calidad del agua obtenida en cada tipo fluvial en la cuenca del Ebro en el año 2005. Percentage of each water quality class in each fluvial type in the Ebro Basin in 2005.

del periodo 1990-2005, hasta estar analizándose en los últimos años un número de estaciones de muestreos que resulta representativo para conocer el estado de la cuenca, refleja el mayor interés y la importancia que se viene concediendo al estudio del estado de las aguas mediante índices biológicos. Esto también se puede deducir por el hecho de que en los últimos años se ha incrementado el número de estudios sobre este tema en diferentes cuencas hidrográficas peninsulares, como por ejemplo la cuenca del Duero (García de Jalón \& González del Tánago, 1986),

Tabla 1. Comparación del valor del índice IASPT hallado en 2005 en cada tipo fluvial de la cuenca del Ebro respecto a la media historica existente. Comparison of the value of the IASPT index found in 2005 in each fluvial type of the Ebro basin, in relation to the existing historical mean value.

\begin{tabular}{lcr}
\hline Ecotipo & Mejora IASPT & Empeora IASPT \\
\hline Ríos mineralizados de baja montaña mediterránea & 79.0 & 21.0 \\
Ríos de montaña mediterránea calcárea & 52.0 & 48.0 \\
Ejes medit. continentales poco mineralizados & 76.5 & 23.5 \\
Ejes medit. continentales mineralizados & 100.0 & 0.0 \\
Grandes ejes en ambiente mediterráneo & 54.6 & 45.4 \\
Ríos de montaña húmeda calcárea & 50.0 & 50.0 \\
Ríos de alta montaña & 14.3 & 85.7 \\
\hline
\end{tabular}


Ríos mineralizados de baja montaña mediterránea

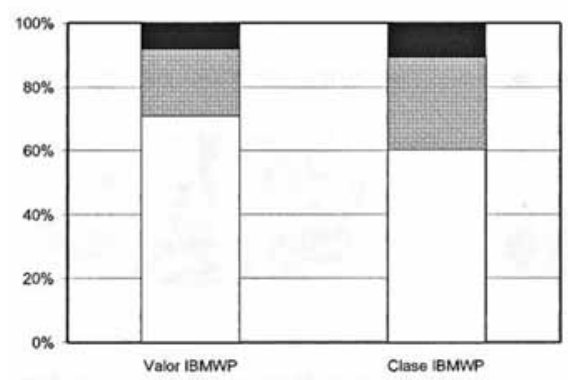

Ejes mediterráneos continentales poco mineralizados

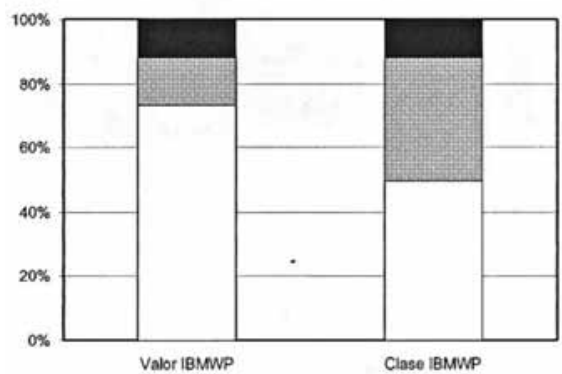

Grandes ejes en ambiente mediterráneo

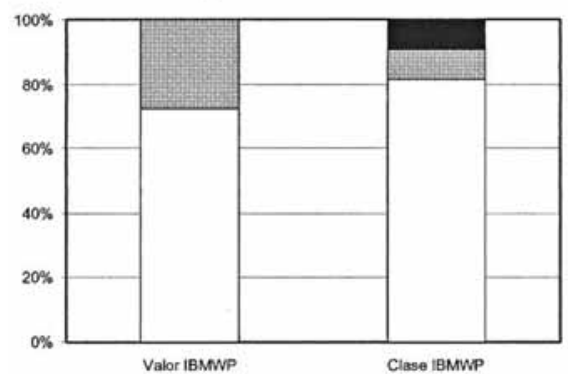

Rios de alta montaña

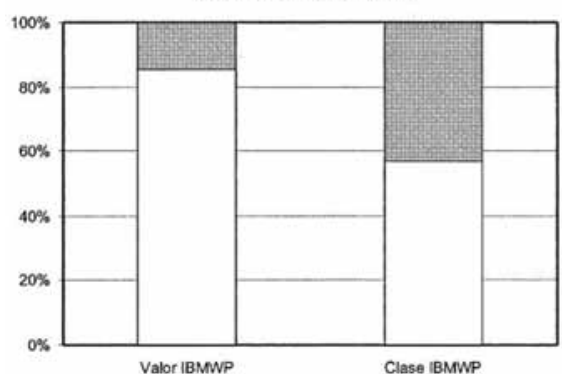

Ríos de montaña mediterránea calcárea

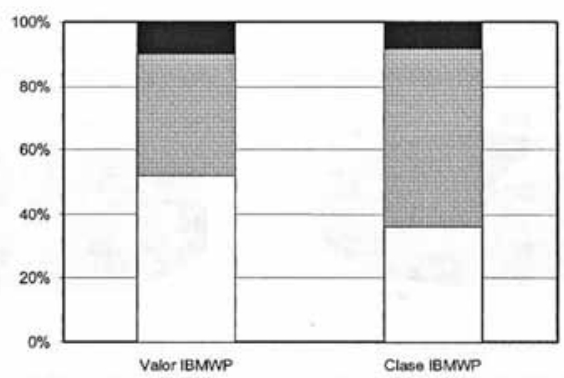

Ejes mediterráneos continentales mineralizados

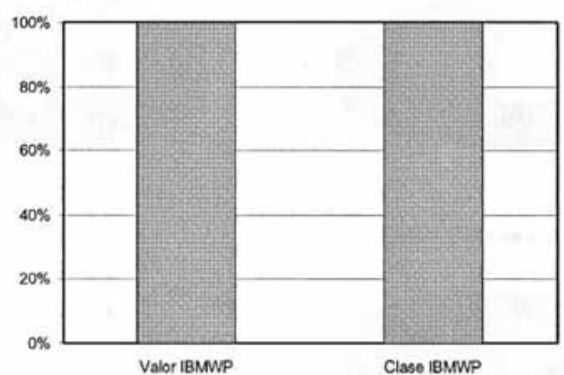

Rios de montaña húmeda calcárea

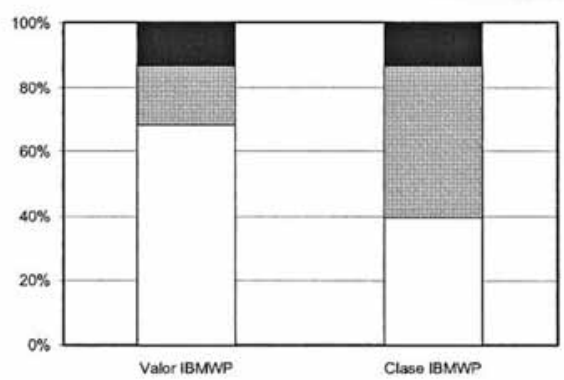

Valor IBMWP

Clase IBMWP

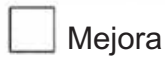

Similar

Empeoramiento

Figura 6. Comparación del valor del índice IBMWP y de la clase de calidad hallada en 2005 en cada tipo fluvial de la cuenca del Ebro respecto a la media histórica existente. Comparison of the IBMWP index value and quality class found in 2005 in each fluvial type, in relation to the historical mean value.

los ríos del País Vasco (Rallo et al., 1990; Gobierno Vasco, 1995), los ríos catalanes (Prat et al.,2000), la cuenca del Júcar (Martínez Mas et al., 2004) o la propia cuenca del Ebro (Gil Quilez et al., 2001; Oscoz et al., 2004; 2005; 2007). Por otra parte, la mejora detectada en el perio- 
do analizado respecto al porcentaje de estaciones que alcanzaron una calidad "Buena" o "Muy Buena” indicaría que los programas y estrategias que se han desarrollado en los últimos años de cara a minimizar la contaminación y la cantidad de vertidos, favoreciendo una mayor concienciación social sobre la necesidad de proteger los sistemas acuáticos, pueden estar empezando a dar su fruto. Así por ejemplo, el aumento en este periodo del número de estaciones depuradoras de aguas residuales en funcionamiento y la población cuyas aguas fecales eran tratados por dichas depuradoras (Tabla 2) sería un reflejo de estas mejoras introducidas en la cuenca del Ebro que estarían favoreciendo la recuperación de la calidad del agua y el estado ecológico de los sistemas fluviales. La aparente gran mejoría que parecería detectarse en el periodo 1998-2000 estaría condicionada porque dichos años el estudio de macroinvertebrados se realizó en un reducido número de estaciones de muestreo, lo que afectaría a la validez de los resultados como reflejo del estado de toda la cuenca. Es por ello que no se puede deducir que el conjunto de la cuenca del Ebro empeorara su estado ecológico entre 2001 y 2004 respecto a las campañas pre-

Tabla 2. Número estaciones depuradoras (EDAR) y población con EDAR a lo largo del periodo 1989-2005 en la cuenca del río Ebro. Number of water treatment plants (WTP) and population with WTP in the Ebro River Basin during the period 1989-2005.

\begin{tabular}{ccc}
\hline Año & Estaciones Depuradoras & Población \\
\hline 1989 & 1 & 53217 \\
1990 & 1 & 53217 \\
1991 & 1 & 53217 \\
1992 & 3 & 56359 \\
1993 & 4 & 653831 \\
1994 & 4 & 653831 \\
1995 & 5 & 654007 \\
1996 & 5 & 654007 \\
1997 & 8 & 661510 \\
1998 & 112 & 1247737 \\
1999 & 129 & 1614766 \\
2000 & 142 & 1674430 \\
2001 & 179 & 1922127 \\
2002 & 197 & 1997003 \\
2003 & 203 & 2014576 \\
2004 & 223 & 2086821 \\
2005 & 259 & 2102671 \\
\hline
\end{tabular}

cedentes, sino que más bien se mantuvo en porcentajes similares a los de los años 1997 y anteriores, encontrándose una mejora en los porcentajes de estaciones con un estado ecológico "Bueno" o "Muy Bueno" en el año 2005.

Al diferenciar la evolución histórica existente en los distintos tipos fluviales, y nuevamente considerando aparte el periodo 1998-2000 por el problema antes mencionado, se observa que es en general en tramos localizados en ríos de cabecera o ríos de montaña donde la calidad se ha mantenido en valores altos desde los primeros años. Esto es una situación normal, pues ya se ha descrito que el IBMWP presenta una alta correlación con la altitud y con la calidad del hábitat fluvial (Alba-Tercedor et al., 2002; Pardo et al., 2002), debido a que son en general estos tramos altos los que han sufrido una menor degradación en sus cuencas y riberas y poseen una menor cantidad de vertidos y alteraciones ambientales. En aquellos tipos que engloban a tramos medios y bajos de afluentes del Ebro (como por ejemplo el ecotipo -Rios mineralizados de baja montaña mediterránea-) es donde se observa más claramente la mejora experimentada a lo largo de los años. Esto indicaría que la mejora global en la calidad se ha dado en gran parte de la cuenca, y no es debida a la mejora parcial de áreas localizadas de la cuenca del Ebro, ya que estas zonas medias y bajas también se verían positivamente afectadas por la mejora en tramos más altos. Sin embargo, todavía en el tramo medio-bajo del río Ebro (tipo fluvial -Grandes ejes en ambiente mediterráneo-), y en el tramo bajo del río Jalón (tipo fluvial -Ejes continentales mediterráneos mineralizados-) la mejora de la calidad podría no estar consolidada o no se ha producido. En el caso de la parte mediabaja del río Ebro, a pesar de haberse incrementado en general la calidad en el periodo de estudio, todavía no se puede considerar que la situación sea la más aceptable, sino que debería aún trabajarse más en mejorar la calidad de este tramo. En el caso del tramo bajo del río Jalón, la calidad no ha mejorado, ni se llegan a alcanzar niveles de calidad Buena, lo que implicaría que en este tramo no se alcanzaría el estado ecológico que la DMA exige. Las causas del mal estado de las aguas en este corto tramo del río Jalón pueden es- 
tar motivadas por los vertidos urbanos, industriales y agrícolas existentes en la zona, unidos al intensivo aprovechamiento hidráulico, fundamentalmente para riego, que existe en esta subcuenca.

Se debe apuntar también que parte de la variación de los datos hallados a lo largo del periodo de estudio podrían parcialmente estar relacionados con el hecho de que los responsables del muestreo han cambiado a lo largo de los años, así como con el hecho que desde los años iniciales del estudio la metodología ha podido modificarse ligeramente, hasta haberse consolidado con el establecimiento del protocolo de muestreo específico (Jáimez-Cuellar et al., 2002).

\section{Calidad del agua en el año 2005}

En el año 2005 el porcentaje de tramos que alcanzaron el objetivo de estado ecológico "Bueno" o superior que exige la DMA fue casi del $80 \%$, lo que se puede interpretar como un dato muy positivo. Además este notable porcentaje de estaciones con estado ecológico "Bueno" se debe valorar de manera aún más positiva si se tiene en cuenta que el 2005 fue un año de sequía, lo que a priori podía haber acentuado los problemas de contaminación en los ríos, debido al menor caudal existente en general en ellos. También se puede calificar como positivo el hecho de que más del $65 \%$ de las estaciones analizadas tuvieran un valor del índice mayor que su media histórica, ya que esto sería reflejo, o bien de un aumento en la riqueza de taxones en el tramo, o bien de un cambio en el porcentaje de taxones con alto valor en el índice (taxones con mayores requerimientos ecológicos), lo cual se ve también en la mejora de los valores del índice IASPT. Ambas posibilidades indicarían que los tramos analizados han tenido una recuperación o un incremento de su estado ecológico, lo que también se puede inferir por el elevado número de estaciones (casi el $50 \%$ ) que alcanzaron en el año 2005 una calidad mayor que la correspondiente a su media histórica. Podría parecer paradójico que habiéndose mejorado el valor del índice en más del $65 \%$ de las estaciones, sólo en un $50 \%$ de ellas se mejore la clase de calidad, pero esto se debe a que un aumento en el valor del índice no implica una mejora en la clase de calidad. Es más, se puede tener históricamente un valor de índice indicativo de clase “Muy Buena”, y mejorar dicho valor, pero no se puede mejorar la clase de calidad, pues ya se está en el nivel de calidad más alto.

El análisis del estado ecológico en el año 2005 diferenciando tipos fluviales volvió a mostrar que son los ríos correspondientes a tramos de cabecera o ríos de montaña los que presentan mayor porcentaje de puntos con calidad "Muy Buena". Sin embargo, la calidad alcanza el nivel que la DMA exige en la mayor parte de los tipos fluviales analizados, no alcanzándose únicamente en el tipo correspondiente al tramo bajo del río Jalón, al que ya nos hemos referido en el apartado anterior. Destacan sobre todo los resultados hallados en el tipo -Grandes ejes en ambiente mediterráneo-, correspondiente a gran parte del eje del Ebro, con un $83 \%$ de estaciones de calidad "Buena". Este hecho estaría motivado por la buena calidad del agua hallada en la mayor parte de las estaciones en este tramo en el año 2005. Solamente la calidad se mantuvo en niveles bajos por debajo de núcleos urbanos e industriales importantes (como Zaragoza) y por debajo del embalse de Flix. Posiblemente la buena calidad que el río Ebro mantuvo en 2005 en casi todo su recorrido pudo estar influido por el hecho de que para mantener el caudal de Verano en niveles similares a los de pasados años en el 2005 se desembalsaba más agua del Embalse del Ebro, la cual tendría buena calidad. De esta forma, y respecto a años anteriores, el caudal circulante en el río Ebro estaría compuesto en mayor medida o principalmente por agua de mejor calidad, lo que habría provocado los buenos resultados hallados con los índices bióticos.

La mejoría en el valor del índice IASPT en la mayoría de los ecotipos también estarían mostrando que la mejora del estado de las aguas en el año 2005 respecto a la media histórica se estaría produciendo en el conjunto de la cuenca. Sin embargo, y al igual que ocurría con el índice IBMWP, los porcentajes de aumento en el índice fueron mayores en ecotipos localizados en tramos medios y bajos del río Ebro y sus afluentes, especialmente -Ríos mineralizados de baja montaña mediterránea- y -Ejes mediterráneos 
continentales poco mineralizados-. Destaca también que en el ecotipo -Ejes mediterráneos continentales mineralizados-, pese a no mejorarse el IBMWP si se ha hallado una mejora en el IASPT, lo que indicaría que le mejora no ha sido sustancial, y que sigue habiendo factores condicionantes respecto a la calidad. En cuanto al mayor descenso registrado para el IASPT en el ecotipo -Ríos de alta montaña-, aunque debería analizarse más profundamente para comprobar si el aumento de las presiones humanas en esta zona puede estar empezando a afectar a los sistemas fluviales, no parece preocupante, puesto que el índice IBMWP no mostró empeoramiento. Además, al no existir todavía rangos establecidos para el índice IASPT no es seguro que los descensos observados pudieran resultar significativos. Estos resultados deberían analizarse más detalladamente en estudios futuros, especialmente ahora que los protocolos y la metodología de muestreo se han normalizado.

Estos resultados muestran que, en el periodo analizado, se ha dado en la cuenca del Ebro una mejora general de los valores del IBMWP hasta el año 2000, con un periodo de mantenimiento de la situación hasta una nueva mejora en el año 2005, lo que reflejaría una mejora paulatina en el estado de las aguas. Hay que señalar sin embargo que todavía quedan aspectos a mejorar para poder alcanzar el ansiado objetivo de buen estado ecológico que la DMA exige para nuestras aguas. Un análisis más detallado de los tramos que no cumplen el nivel de calidad exigido nos debe llevar a determinar las causas que lo motivan, pudiendo así plantearse el programa de medidas necesario para conseguir el buen estado, en el caso de que ese mal estado se deba a causas no naturales.

\section{AGRADECIMIENTOS}

Nuestro agradecimiento a la Oficina de Planificación Hidrológica de la Confederación Hidrográfica del Ebro por facilitarnos los datos de los índices bióticos existentes en la cuenca del Ebro para el periodo 1990-2002, así como por proporcionarnos los datos sobre la evolución en el núme- ro de depuradoras y población con tratamiento de aguas en la cuenca del Ebro. Asimismo nuestro agradecimiento a Arantxa Imaz, Ainhoa Agorreta y Maite Martínez Aldaya por su colaboración en alguno de los muestreos realizados en los años 2004 y 2005.

\section{BIBLIOGRAFÍA}

ALBA-TERCEDOR, J. 1996. Macroinvertebrados acuáticos y calidad de las aguas de los ríos. IV Simposio del Agua en Andalucía (SIAGA), Almeria, España, vol. II: 203-213.

ALBA-TERCEDOR, J., P. JÁIMEZ-CUÉLLAR, M. ÁlVAREZ, J. AVILÉS, N. BONADA, J. CASAS, A. MELLADO, M. ORTEGA, I. PARDO, N. PRAT, M. RIERADEVALL, S. ROBLES, C. E. SÁINZ-CANTERO, A. SÁNCHEZ-ORTEGA, M. L. SUÁREZ, M. TORO, M. R. VIDAL-ABARCA, S. VIVAS y C. ZAMORA-MUÑOZ. 2002. Caracterización del estado ecológico de ríos mediterráneos ibéricos mediante el índice IBMWP (antes BMWP'). Limnetica, 21(3-4): 175-185

ALBA-TERCEDOR, J. y A. SÁNCHEZ-ORTEGA. 1988. Un método rápido y simple para evaluar la calidad biológica de las aguas corrientes basado en el de Hellawell (1978). Limnetica, 4: 51-56.

D.O.C.E. 2000. Directiva 2000/60/CE del Parlamento Europeo y del Consejo de 23 de octubre de 2000 por la que se establece un marco comunitario de actuación en el ámbito de la política de aguas. D.O.C.E. L 327 de 22.12.00. 69 pp.

DOLÉDEC, S., J. M. OLIVIER \& B. STATZNER. 2000. Accurate description of the abundance of taxa and their biological traits in stream invertebrate communities: effects of taxonomic and spatial resolution. Archiv Hydrobiol., 148(1): 25-43.

GARCÍA DE JALÓN, D. y M. GONZÁLEZ DEL TÁNAGO. 1986. Métodos biológicos para el estudio de la calidad de las aguas. Aplicación a la Cuenca del Duero. ICONA Monogr., 45: 1-244.

GARCÍA DE JALÓN, D., M. GONZÁLEZ DEL TÁNAGO y M. GARCÍA DE VIEDMA. 1980. Importancia de los insectos en los métodos biológicos para el estudio de la calidad de las aguas: necesidad de su conocimiento taxonómico. Graellsia, 35-36: 143-148.

GIL QUILEZ, M. J., A. PALAU y C. FERNÁNDEZ MANZANAL. 2001. Calidad biológica (BMWP') 
de las aguas del rio Cinca (Huesca). Limnetica, 20(1): 107-113.

GOBIERNO VASCO. 1995. Red de vigilancia de la calidad de las aguas y del estado ambiental de los ríos de la C.A.P.V. Dpto. de Urbanismo, Vivienda y Medio Ambiente. Viceconsejería de Medio Ambiente del Gobierno Vasco. Vitoria. 178 pp.

GRACA, M. A. S., C. N. COIMBRA \& L. M. SANTOS. 1995. Identification level and comparison of biological indicators in biomonitoring programs. Cienc. Biol. Ecol. Syst., 15(1/2): 9-20.

JÁIMEZ-CUELLAR, P., S. VIVAS, N. BONADA, S. ROBLES, A. MELLADO, M. ÁlVAREZ, J. AVILÉS, J. CASAS, M. ORTEGA, I. PARDO, N. PRAT, M. RIERADEVALL, C. E. SÁINZCANTERO, A. SÁNCHEZ-ORTEGA, M. L. SUÁREZ, M. TORO, M. R. VIDAL-ABARCA, C. ZAMORA-MUÑOZ y J. ALBA-TERCEDOR. 2002. Protocolo GUADALMED (PRECE). Limnetica, 21(3-4): 187-204.

MARTÍNEZMAS, J.F., E. CORRECHER, A. PIÑÓN, M. A. MARTÍNEZ MURO, y A. M. PUJANTE. 2004. Estudio del estado ecológico de los ríos de la cuenca hidrográfica del Júcar (España) mediante el índice BMWP'. Limnetica, 23(3-4): 331-346.

OSCOZ, J., F. CAMPOS, y M. C. ESCALA. 2004. Calidad biológica de las aguas del río Larraun (Navarra) (1996-1997). Ecología, 18: 11-20.
OSCOZ, J., P. M. LEUNDA., R. MIRANDA, y M. C. ESCALA. 2005. Calidad biológica de las aguas en el río Erro (Navarra, N España) (2001-2002). Ecología, 19: 59-74.

OSCOZ, J., J. GOMÀ, L. ECTOR, J. CAMBRA, M. PARDOS y C. DURÁN. 2007. Estudio comparativo del estado ecológico de los ríos de la cuenca del Ebro mediante macroinvertebrados y diatomeas. Limnetica, 26(1): 143-158.

PARDO, I., M. ÁlVAREZ, J. CASAS, J. L. MORENO, S. VIVAS, N. BONADA, J. ALBATERCEDOR, P. JÁIMEZ-CUELLAR, G. MOYÀ, N. PRAT, S. ROBLES, M. L. SUÁREZ, M. TORO y M. R. VIDA-ABARCA. 2000. El hábitat de los ríos mediterráneos. Diseño de un índice de diversidad de hábitat. Limnetica, 21(3-4): 115-133.

PRAT, N., A. MUNNÉ, C. SOLÀ, R. CASANOVAS, M. VILA, N. BONADA, J. JUBANY, M. MIRALLES, M. PLANS y M. RIERADEVALL. 2000. La qualitat ecològica del Llobregat, el Besòs, el Foix i la Tordera. Estudis de la qualitat ecològica dels rius 10. Area Medi Ambient, Diputació de Barcelona. 163 pp.

RALLO, A., B. GARCÍA DE BIKUÑA, E. RICO y M. SEVILLANO. 1990. Caracterización hidrobiológica de la red fluvial de Álava y Guipúzcoa. Informe científico. Gobierno Vasco, Universidad del País Vasco, 70 pp. 